\title{
UMA NOVA ROTA TECNOLÓGICA PARA A PRODUÇÃO DE HIDRÓXIDO DE LÍTIO A PARTIR DE MINÉRIO DE ESPODUMÊNIO
}

\author{
MOREIRA, G. C. $^{1}$, GONÇALVES, C. C. $^{1}$, FRANCA, S. C. A $^{1}$, BRAGA, P. F. A ${ }^{1}$ \\ ${ }^{1}$ Centro de Tecnologia Mineral - CETEM, Coordenação de Processamento e Tecnologias Minerais - COPTM. \\ pbraga@cetem.gov.br
}

Submetido 13/11/2017 - Aceito 20/11/2017

DOI: $10.15628 /$ holos.2017.6534

\section{RESUMO}

O lítio é um metal de grande importância para matriz energética mundial devido a sua aplicação na produção de baterias para veículos elétricos e equipamentos de portabilidade (celulares, notebooks, smartphones, ferramentas, etc.). Os produtos de lítio de maior interesse econômico são o carbonato e o hidróxido de lítio, sendo o hidróxido de alta pureza o composto chave principal dos novos projetos de lítio em desenvolvimento no mundo, função de seu uso na fabricação de catodos para as baterias Li-íon com maior potencial energético, que são utilizadas em veículos elétricos (EVs) e sistemas de armazenamento de energia de grande porte (grid storage). As reservas brasileiras de lítio são provenientes de pegmatitos litiníferos, mais especificamente os minerais espodumênio e ambligonita. A indústria nacional utiliza a rota ácida para a produção de carbonato de lítio, a qual está estabelecida desde a década de 40 , mas com custo
\end{abstract}

operacional alto, devido à utilização de barrilha e ácido sulfúrico, insumos caros e importados e que são descartados durante a obtenção do hidróxido. Com o intuito aumentar a sustentabilidade do processo e a competitividade da indústria brasileira no mercado global, fornecendo um produto de maior pureza e menor custo, percebeu-se a necessidade do desenvolvimento de novas rotas de obtenção deste composto, o hidróxido. Foi desenvolvida uma rota alternativa, partindo-se das etapas iniciais do processo ácido e concluindo-se com a rota alcalina, cujo princípio baseia-se na caustificação do sulfato de lítio, até a obtenção do hidróxido de lítio. A recuperação metalúrgica de lítio, nessa etapa do processo, foi superior a 98\%. O hidróxido de lítio monohidratado obtido possui uma pureza de 55,07\%, superior a do produto produzido pela indústria nacional.

PALAVRAS-CHAVE: lítio, espodumênio, hidróxido de lítio.

\section{A NEW TECHNOLOGICAL ROUTE FOR PRODUCTION OF LITHIUM HYDROXIDE FROM SPODUMENE ORE}

\begin{abstract}
Lithium is a metal of great importance to the global energy market due to its application in the production of batteries for electric vehicles and portable devices (cell phones, notebooks, smartphones, tools, etc.). The lithium compounds, which have an elevated economic value, are the lithium carbonate and the lithium hydroxide, being the lithium hydroxide with high purity the main compound considered in new projects around the world especially because its uses in the production of cathodes for Li-ion batteries with higher energy potential such as NCA (Lithium Nickel Cobalt Aluminum Oxide), NMC (Lithium Nickel Manganese Cobalt Oxide) and LFP (Lithium Iron Phosphate) which are used in automotive batteries (EVs) and huge electrical storage systems (grid storage). The Brazilian lithium reserves are originated from pegmatites, specifically spodumene mineral and amblygonite. The national industry uses the acidic route for the lithium hydroxide production that was established since the 40's but with a high operating costs due to the use of soda ash and sulfuric acid, expensive and imported inputs. In order to increase the sustainability of the process and the competitiveness of the Brazilian industry in the global market, providing a higher purity and low cost product, it was realized the necessity of establishing new
\end{abstract}

routes for obtaining that compound, the lithium hydroxide. It was developed an alternative route for obtaining the lithium hydroxide, starting with the initial stages of the acid route, and ending up with the alkaline route, whose the principle is based on the causticizing of lithium sulfate to obtain lithium hydroxide. The lithium recovery in the metallurgical process was up to $98 \%$. The lithium hydroxide monohydrate obtained has a purity of $55.07 \%$, higher than the national product.

KEYWORDS: lithium; spodumene; lithium hydroxide. 


\section{INTRODUÇÃO}

O elemento lítio (Li) é um metal alcalino com características físicas e químicas distintas. Sua baixa densidade, menor peso molar dentre todos os metais, e elevado potencial eletroquímico, até mesmo quando comparado ao potencial eletroquímico do eletrodo padrão de hidrogênio (Jeppson et al., 1978), torna esse elemento um insumo de imensurável importância para a indústria mundial, principalmente no que concerne o setor de produção baterias.

Os principais compostos derivados do lítio, utilizados na indústria, são o carbonato de lítio $\left(\mathrm{Li}_{2} \mathrm{CO}_{3}\right)$, o hidróxido de lítio $(\mathrm{LiOH})$ e o butil-lítio $\left(\mathrm{C}_{4} \mathrm{H} \mathrm{H}_{9} \mathrm{Li}\right)$. O carbonato de lítio é principalmente utilizado na produção de alumínio e na indústria de vidros e cerâmicas. Já o hidróxido é usado na produção de baterias de íon-Li e graxas lubrificantes. O butil-lítio tem sua aplicação voltada para a área de farmacêuticos e agroquímicos (Braga, França, 2013). Atualmente, a produção destes compostos pode ser proveniente de salmouras, pegmatitos litiníferos, argilas hectoríticas e água do mar. A nível mundial, os compostos litiníferos são normalmente obtidos a partir de salmouras (59\%), como o Salar de Atacama, o Salar del Hombre-muerto e alguns desertos na China. A produção de compostos litiníferos a partir de pegmatitos concentra-se principalmente na Austrália (Moreno, 2013).

Existem duas rotas tecnológicas distintas, sejam a rota ácida, consolidada na indústria nacional, e a rota alcalina, as quais foram desenvolvidas na década de 1940 (Braga e França, 2013). No Brasil, a extração do lítio é feita a partir de pegmatitos, em especial do mineral espodumênio $\left(\mathrm{Li}_{2} \mathrm{O} . \mathrm{Al}_{2} \mathrm{O}_{3} \cdot 4 \mathrm{SiO}_{2}\right)$.

Ambas iniciam-se com a conversão (Equação 1), por tratamento térmico (T $\left.1050^{\circ} \mathrm{C}\right)$, de $\alpha$ espodumênio (espécie in natura) em $\beta$-espodumênio, o qual possui maior friabilidade e reatividade (Medina e El-Nagar, 1984).

$$
\alpha-\mathrm{Li}_{2} \mathrm{O} \cdot \mathrm{Al}_{2} \mathrm{O}_{3} \cdot 4 \mathrm{SiO}_{2} \frac{\sim 1.050^{\circ} \mathrm{C}}{\underline{\Delta}} \rightarrow \beta-\mathrm{Li}_{2} \mathrm{O} \cdot \mathrm{Al}_{2} \mathrm{O}_{3} \cdot 4 \mathrm{SiO}_{2}
$$

Assim, no processo ácido (Figura 1) o $\beta$-espodumênio, após sulfatação com ácido sulfúrico $\left(\mathrm{H}_{2} \mathrm{SO}_{4}\right)$, é lixiviado com água para a formação do sulfato de lítio $\left(\mathrm{Li}_{2} \mathrm{SO}_{4}\right)$ (Equação 2). A solução de sulfato de lítio é purificada de contaminantes ( $\mathrm{Fe}, \mathrm{Al}, \mathrm{Ca}$ e $\mathrm{Mg}$ ) e em seguida é convertida a carbonato de lítio pela reação com o carbonato de sódio $\left(\mathrm{Na}_{2} \mathrm{CO}_{3}\right)$ (Equação 3) (Habashi, 1997).

$$
\begin{gathered}
\beta-\mathrm{Li}_{2} \mathrm{O} \cdot \mathrm{Al}_{2} \mathrm{O}_{3} \cdot 4 \mathrm{SiO}_{2}+\mathrm{H}_{2} \mathrm{SO}_{4} \rightarrow \mathrm{Li}_{2} \mathrm{SO}_{4}+\mathrm{H}_{2} \mathrm{O} \cdot \mathrm{Al}_{2} \mathrm{O}_{3} \cdot 4 \mathrm{SiO}_{2} \\
\mathrm{Li}_{2} \mathrm{SO}_{4}+\mathrm{Na}_{2} \mathrm{CO}_{3} \rightarrow \mathrm{Li}_{2} \mathrm{CO}_{3}+\mathrm{Na}_{2} \mathrm{SO}_{4}
\end{gathered}
$$




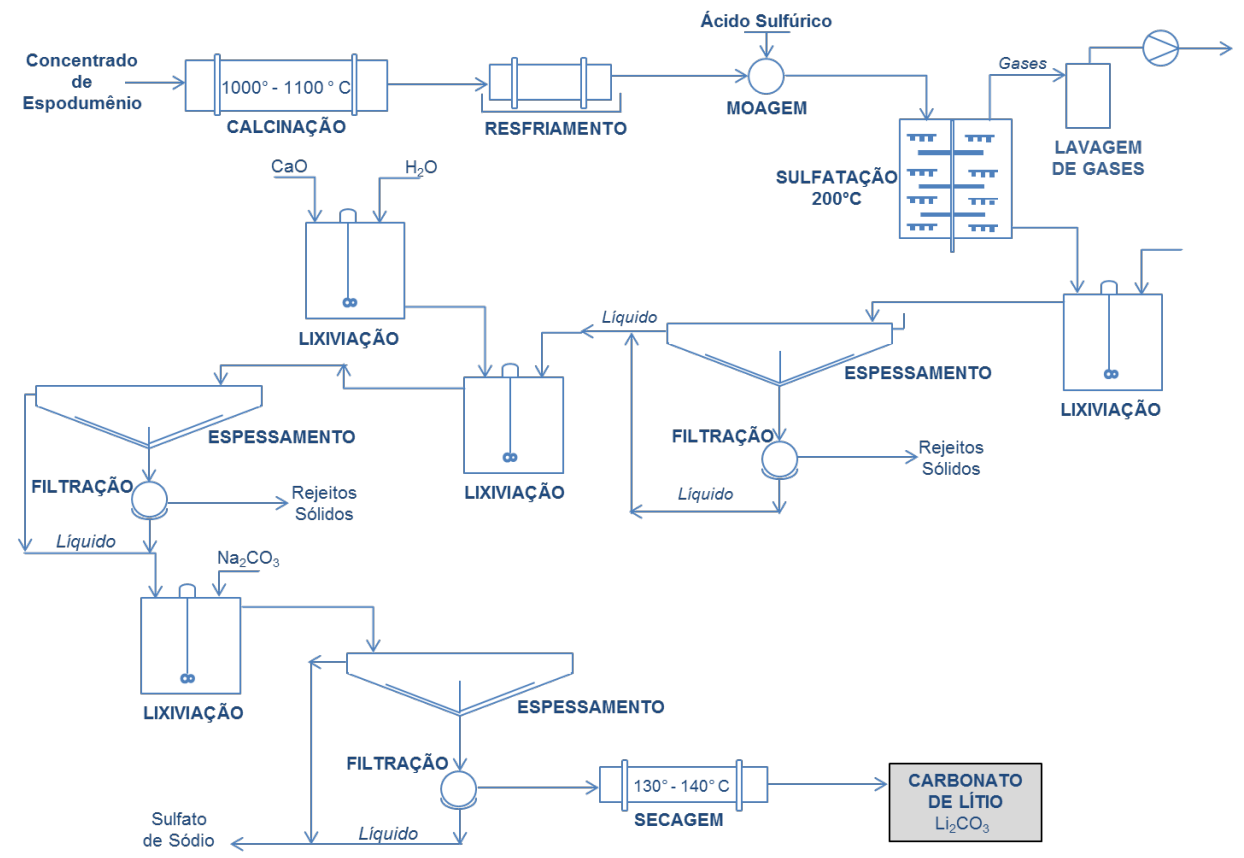

Figura 1. Fluxograma do processo ácido.

No processo alcalino (Figura 2), por sua vez, após o mesmo tratamento térmico supracitado, o $\beta$-espodumênio é caustificado (reação sólido/sólido) com cal ou calcário (Equação 4), dando origem a aluminato de lítio e silicato de cálcio (Braga, França, Silva, Rosales, 2015).

$$
\beta-\mathrm{Li}_{2} \mathrm{O} \cdot \mathrm{Al}_{2} \mathrm{O}_{3} \cdot 4 \mathrm{SiO}_{2}+8 \mathrm{CaO} \rightarrow \mathrm{Li}_{2} \mathrm{O} \cdot \mathrm{Al}_{2} \mathrm{O}_{3}+4\left(2 \mathrm{CaO} \cdot \mathrm{SiO}_{2}\right)
$$

$\mathrm{Na}$ etapa de extração (lixiviação), o excesso de cal é hidrolisado (Equação 5), e posteriormente reage com o aluminato de lítio para formação do hidróxido de lítio (Equação 6) (Almeida, 1973).

$$
\begin{gathered}
8 \mathrm{CaO}+\mathrm{H}_{2} \mathrm{O} \rightarrow \mathrm{Ca}(\mathrm{OH})_{2} \\
\mathrm{Li}_{2} \mathrm{O} \cdot \mathrm{Al}_{2} \mathrm{O}_{3}+\mathrm{Ca}(\mathrm{OH})_{2} \rightarrow 2 \mathrm{LiOH}+\mathrm{CaO} \cdot \mathrm{Al}_{2} \mathrm{O}_{3}
\end{gathered}
$$

A rota ácida apresenta um rendimento (em termos de extração de lítio) superior ao da rota alcalina, porém os insumos utilizados são importados e de elevado custo (Braga e França, 2013). Já os insumos referentes à rota alcalina são à base de óxido de cálcio (cal ou calcário), e facilmente supridos pelo mercado nacional e de baixo custo. Entretanto, devido ao uso do LiOH na fabricação de graxas lubrificantes, o carbonato de lítio obtido (Equação 3) deve ser caustificado para a produção do hidróxido correspondente (Equação 7). 


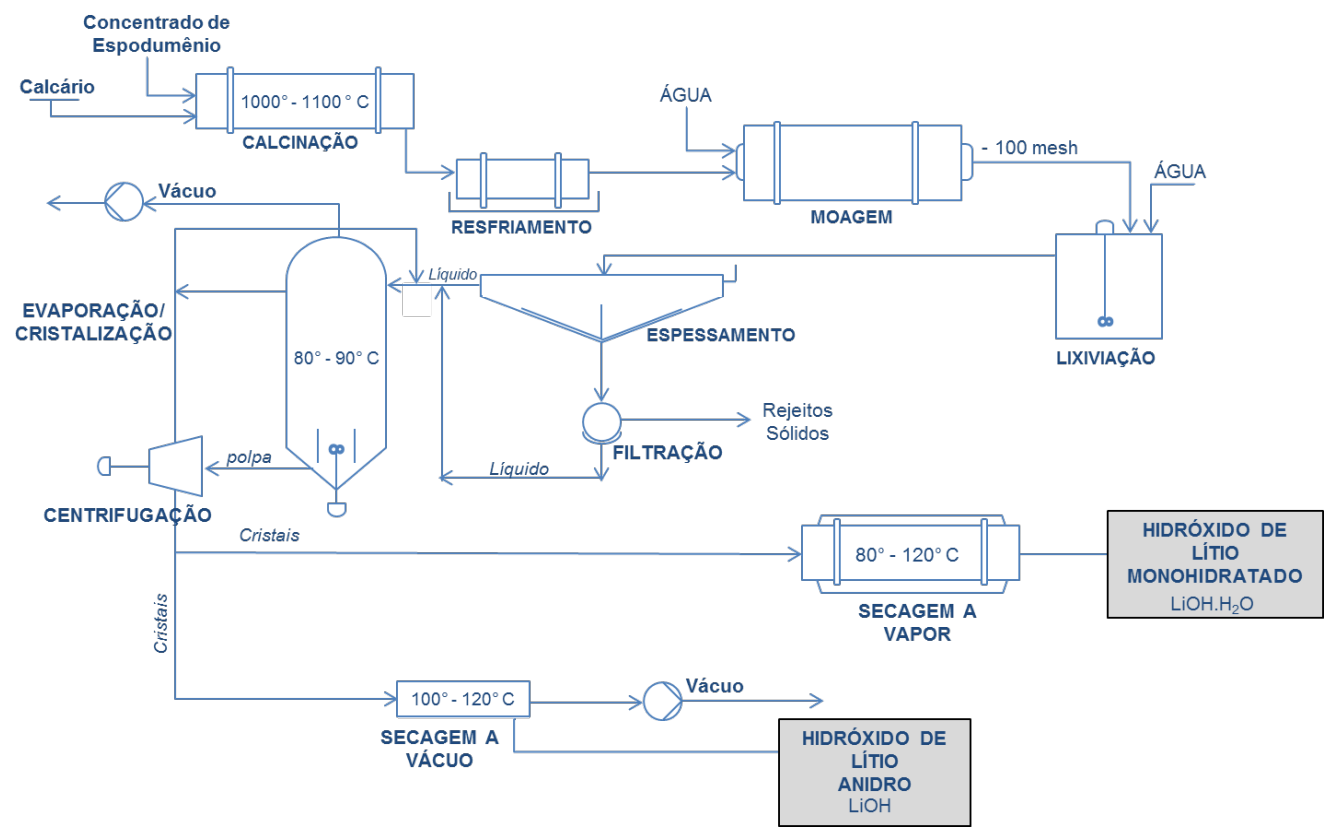

Figura 2. Fluxograma do processo alcalino.

Verifica-se que o processo ácido, utiliza insumos mais caros e importados como o carbonato de sódio (barrilha), que acaba sendo eliminado na caustificação do carbonato e consequente produção do hidróxido de lítio. Percebe-se, então, que o uso da barrilha é necessário para produzir um produto da rota ácida (carbonato), mas que na operação subsequente, produção do hidróxido de lítio (Equação 7) é eliminada sob forma de carbonato de cálcio.

$$
\mathrm{Li}_{2} \mathrm{CO}_{3}+\mathrm{Ca}(\mathrm{OH})_{2} \rightarrow \mathrm{LiOH}+\mathrm{CaCO}_{3}
$$

A Tabela 1 mostra algumas características diferenciais entre as rotas de processo ácida e alcalina para extração de lítio de pegmatitos, a partir do mineral espodumênio.

Tabela 1 - Comparativo entre as rotas ácida e alcalina.

\begin{tabular}{|c|c|}
\hline Rota ácida & Rota alcalina \\
\hline - Maior rendimento em termos de extração de Li & - Menor rendimento em termos de extração \\
\hline $\begin{array}{l}\text { - Maior produtividade, relação } \mathrm{Li}_{2} \mathrm{O}: \mathrm{H}_{2} \mathrm{SO}_{4} \\
\text { estequiométrica ( } 30 \% \text { de ácido em excesso); }\end{array}$ & $\begin{array}{l}\text { - Menor produtividade, devido a relação } \\
\text { espodumênio:cal, 1:2; }\end{array}$ \\
\hline $\begin{array}{l}\text { - Uso de } 2 \text { o forno para sulfatação e geração de gases de } \\
\mathrm{SO}_{2} / \mathrm{SO}_{3}\end{array}$ & - Utilização de um único forno calcinador; \\
\hline - Custo elevado, devido ao uso de barrilha importada; & $\begin{array}{l}\text { - Baixo custo devido ao uso de cal ou calcário } \\
\text { (produtos nacionais); }\end{array}$ \\
\hline $\begin{array}{l}\text { - Produto final é o } \mathrm{Li}_{2} \mathrm{CO}_{3} \text {, sem mercado atualmente no } \\
\text { Brasil; } \\
\text { - Uso adicional de cal para obtenção do } \mathrm{LiOH} . \mathrm{H}_{2} \mathrm{O} \text {. }\end{array}$ & $\begin{array}{l}\text { - Produto final é o } \mathrm{LiOH} . \mathrm{H}_{2} \mathrm{O} \text {, de grande } \\
\text { mercado no segmento de graxas. }\end{array}$ \\
\hline
\end{tabular}

Em vista dessas características dos processos citados uma nova rota foi proposta com objetivo de reduzir o custo de produção do hidróxido de lítio, por este ser um produto de maior uso no mercado nacional e mundial, seja na fabricação de graxas automotivas ou na produção de baterias de Li-íon. 


\section{METODOLOGIA}

\subsection{Amostras, reagentes, materiais e equipamentos}

Inicialmente foi preparada uma solução de $100 \mathrm{~g} / \mathrm{L}$ de sulfato de lítio $\left(\mathrm{Li}_{2} \mathrm{SO}_{4}\right)$ a partir da reação entre o carbonato de lítio $\left(\mathrm{Li}_{2} \mathrm{CO}_{3}\right)$ com pureza de $98,50 \%$ e o ácido sulfúrico concentrado $\left(\mathrm{H}_{2} \mathrm{SO}_{4}\right), 98 \%(\mathrm{p} / \mathrm{p})$, ambos de grau técnico e produzidos nacionalmente (Equação 8).

$$
\mathrm{Li}_{2} \mathrm{CO}_{3}+\mathrm{H}_{2} \mathrm{SO}_{4} \rightarrow \mathrm{Li}_{2} \mathrm{SO}_{4}+\mathrm{CO}_{2}+\mathrm{H}_{2} \mathrm{O}
$$

Como fonte de cálcio, foi utilizado o hidróxido de cálcio $\left[\left(\mathrm{Ca}(\mathrm{OH})_{2}\right]\right.$, com pureza de 95,0\%, da VETEC. No desenvolvimento deste processo foram utilizados os seguintes materiais e equipamentos: provetas graduadas diversas; béqueres diversos; béquer de aço inox 2L; kitassato com funil büchner; placa de aquecimento com agitação magnética; agitador mecânico; balança analítica e semi-analítica e medidores de $\mathrm{pH}$.

\subsection{Desenvolvimento experimental}

Após o estudo das rotas tecnológicas existentes consolidadas para a extração de lítio de pegmatitos (Garrett, 2004), propõe-se um processo alternativo, no qual se utiliza a etapa inicial do processo ácido e conclui-se com a etapa final do processo alcalino. Neste caso o produto final é o hidróxido de lítio, de maior mercado no Brasil (Figura 3).

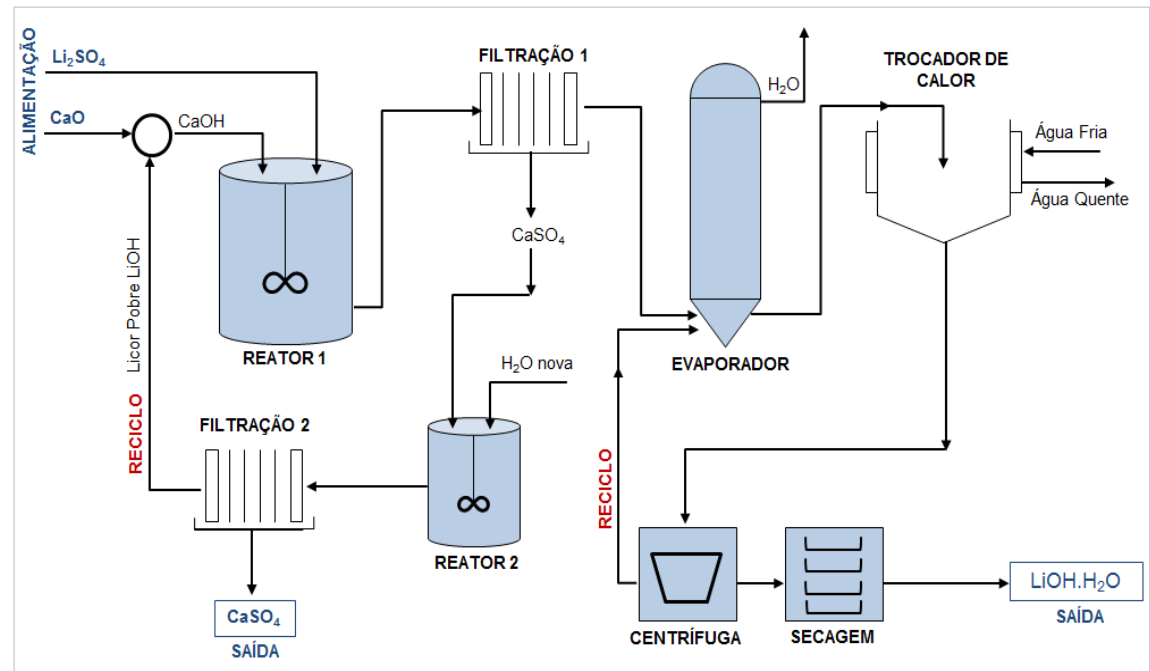

Figura 3. Fluxograma do procedimento experimental desenvolvido

Uma solução de sulfato de lítio $\left(\mathrm{Li}_{2} \mathrm{SO}_{4}\right)$ é caustificada com uma suspensão de $\mathrm{Ca}(\mathrm{OH})_{2}$, em proporção estequiométrica (Equação 7), em reator de aço inox agitado mecanicamente. A reação é realizada na temperatura de $90^{\circ} \mathrm{C}$, para maior atividade reacional, durante 30 minutos, sob agitação vigorosa. Tem-se como produto da reação uma suspensão de hidróxido de lítio com sulfato de cálcio (gesso) precipitado.

$$
\mathrm{Li}_{2} \mathrm{SO}_{4}+\mathrm{Ca}(\mathrm{OH})_{2} \rightarrow 2 \mathrm{LiOH}_{(\mathrm{l})}+\mathrm{CaSO}_{4(\mathrm{~s})}
$$

Após a etapa reacional, a suspensão de hidróxido de lítio com sulfato de cálcio é filtrada a vácuo. O filtrado gerado é uma solução de hidróxido de lítio com concentração de $60 \mathrm{~g} / \mathrm{L}$. $\mathrm{A}$ 
solução de LiOH é então concentrada por evaporação até o limite de saturação (165 g/L), sendo em seguida resfriada a temperatura ambiente e cristalizada sob forma de $\mathrm{LiOH} . \mathrm{H}_{2} \mathrm{O}$. Os cristais de sulfato de lítio são separados da solução mãe por filtração ou centrifugação e secos sob temperatura controlada. A solução mãe, $c o m ~ 25 \mathrm{~g} / \mathrm{L}$ de $\mathrm{LiOH}$, realimenta a etapa de evaporação.

Durante a etapa de reação, a relação sólido:líquido (hidróxido de cálcio:sulfato de lítio) foi de 1:2. Relações inferiores aumentam a rigidez da polpa, dificultando a reação e relações superiores geram uma solução bastante diluída de hidróxido de lítio.

O controle do processo foi realizado com as técnicas de espectrofotometria de chama (fotômetro de chama Analyser 910), espectrometria de absorção atômica (espectrômetro de absorção atômica Varian 55B SpectrAA) e analisador de enxofre e carbono (LECO SC 632 Analyser). A análise de Li foi realizada no espectrômetro de absorção atômica, após solubilização da amostra por meio de solubilização ácida com $\mathrm{H}_{2} \mathrm{SO}_{4}$ e HCl .

\section{RESULTADOS E DISCUSSÃO}

O balanço de metalúrgico de lítio do processo desenvolvido foi calculado em função da recuperação de lítio (\%Li) contido nas correntes de alimentação, reciclo e saídas (Figura 4).

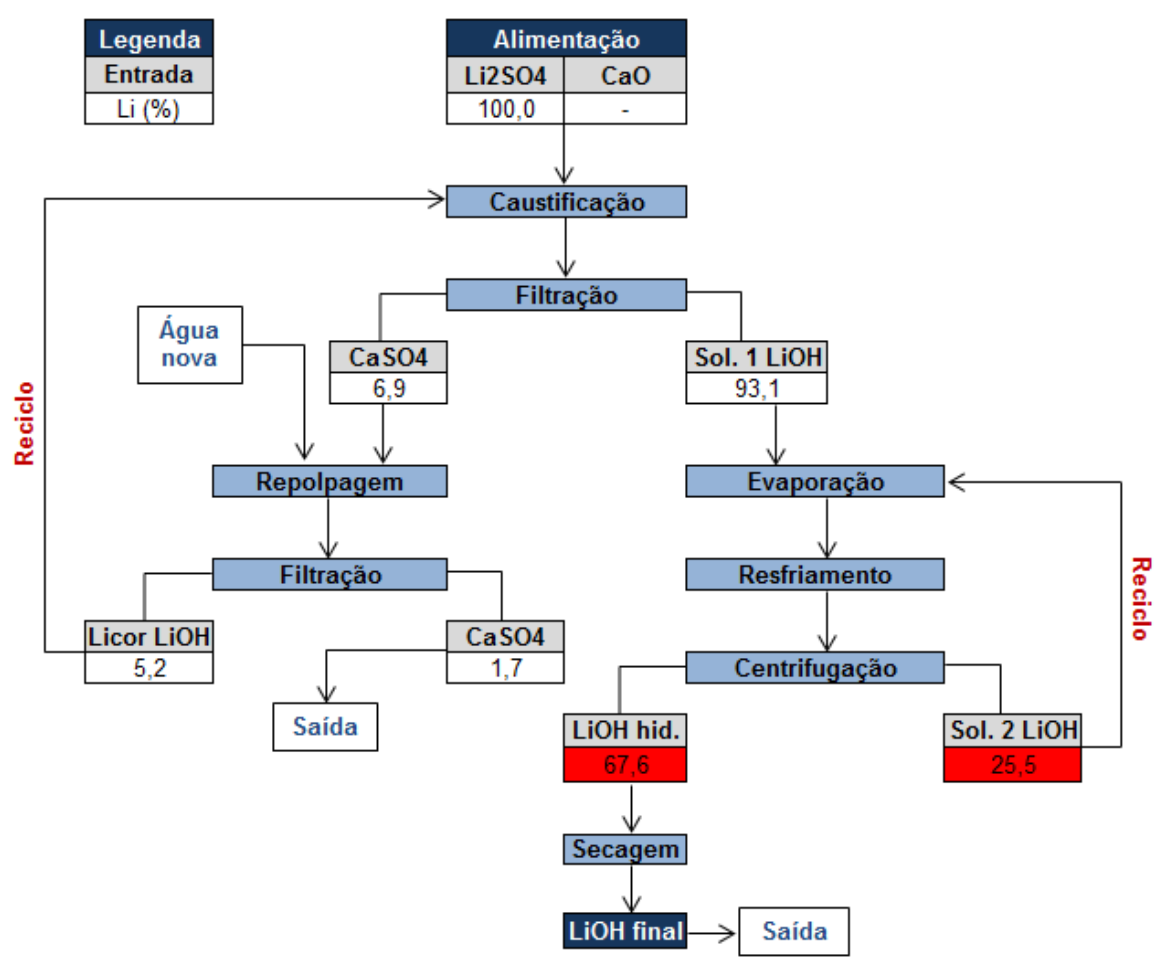

Figura 4. Balanço metalúrgico de lítio do processo desenvolvido.

A recuperação metalúrgica de lítio foi superior a 98\%, nesta etapa hidrometalúrgica. É observada uma perda de $2 \%$ de lítio na corrente de sulfato de cálcio (gesso) repolpado, decorrente de sulfato de lítio não reagido ou retido nas partículas de gesso.

A análise química do hidróxido de lítio apresentou, em termos de pureza, um teor de $55,07 \%$, que é superior ao do produto nacional ( $54,7 \%$ ), porém, inferior ao de um produto de grau 
bateria (56,5\%). A Figura 5 mostra uma comparação entre as purezas dos produtos: (i) técnico nacional; (ii) desenvolvido pela nova rota de processo e; (iii) um produto típico grau bateria.

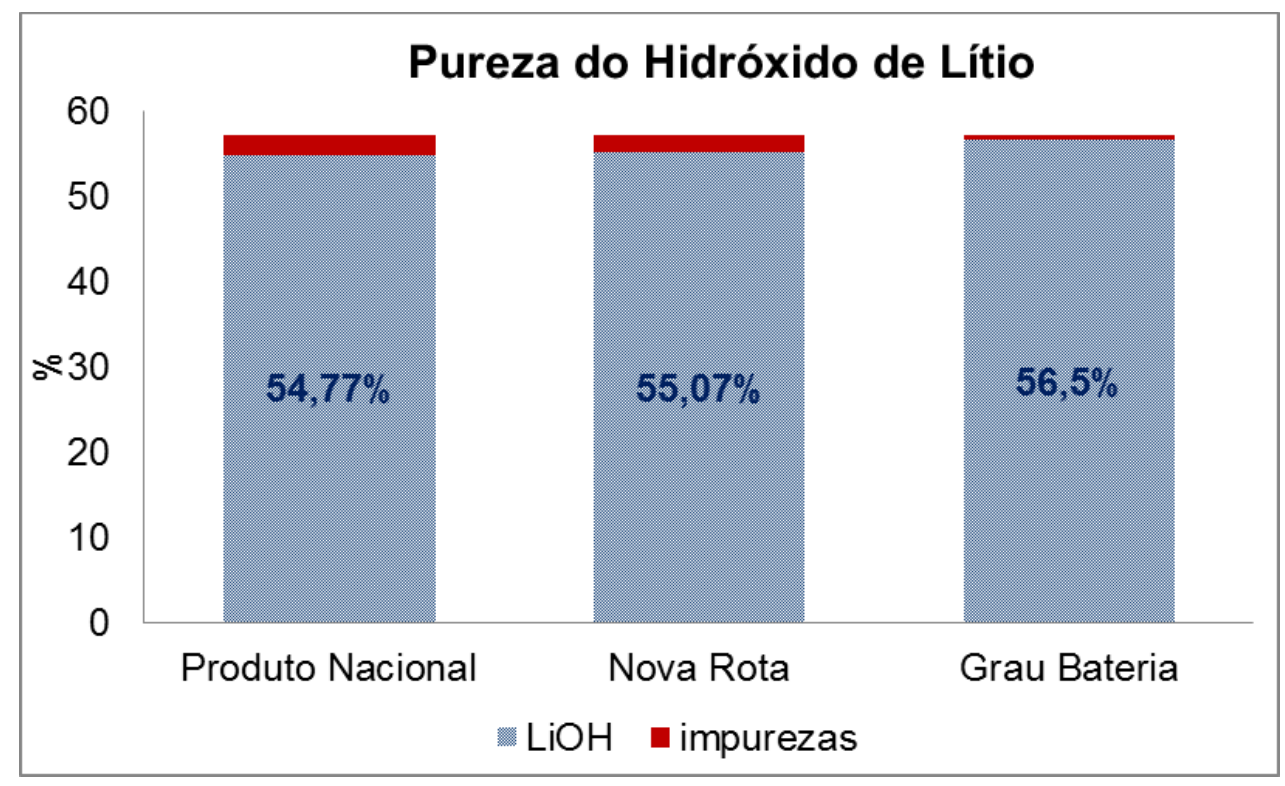

Figura 5. Pureza do hidróxido de lítio (produto nacional, nova rota, grau bateria).

Em relação ao teor de impurezas (íon sulfato, íon carbonato e cálcio) nos hidróxidos de lítio, o somatório das mesmas é de 2,37\%, 2,07\% e 0,64\% para os produtos técnico nacional, desenvolvido pela nova rota de processo e um produto típico de grau bateria, respectivamente. Os teores de íons sulfato e de cálcio (principais contaminantes) estão diretamente associados à solubilidade do gesso, que apesar de baixa $\left(1,84 \mathrm{~g} / \mathrm{L}\right.$ a $\left.90^{\circ} \mathrm{C}\right)$ ainda é significativa para efeito de contaminação do hidróxido. A presença de íons carbonato é devido à carbonatação do hidróxido de lítio pelo gás carbônico da atmosfera, muito comum em processos equivalentes com sodacloro.

Pode-se inferir que a nova rota proposta demonstra resultados coerentes e promissores para novas pesquisas, agora no sentido de purificação/eliminação de impurezas (refino) do hidróxido de lítio. O sucesso da "fusão" dos dois processos consolidados de extração de lítio, a rota ácida e a alcalina, não se deve apenas à ratificação da possibilidade de reação do sulfato de lítio e do hidróxido de cálcio, mas à redução de custos operacionais, devido ao uso de carbonato de sódio, que é um insumo importado.

\section{CONCLUSÕES}

Com base nos resultados do processo desenvolvido, concluiu-se que a mesma apresenta um menor custo operacional devido ao uso cal na caustificação direta da solução de sulfato de lítio, além da eliminação do uso do carbonato de sódio. Este processo apresenta uma alta recuperação de lítio na etapa hidrometalúrgica, superior a $98 \%$. 
O hidróxido de lítio monohidratado $\left(\mathrm{LiOH}_{\mathrm{H}} \mathrm{H}_{2} \mathrm{O}\right)$ produzido apresentou pureza de $55,07 \%$, superior à do produto nacional que é de $54,7 \%$, porém inferior à de um produto de grau bateria, que é de $56,5 \%$.

Novos estudos estão sendo realizados para aumento da pureza do hidróxido de lítio produzido por esse novo processo, de forma a enquadrá-lo em produto grau bateria.

\section{REFERÊNCIAS}

Almeida, A. (1973). Lítio: processos de obtenção, aplicações e perspectivas de produção no Brasil (1st ed.).

Braga, P., \& França, S. (2013). Série Estudos e Documentos 81 - Lítio: Um Mineral Estratégico.. Rio de Janeiro: CETEM/MCTI.

BRAGA, P., França, S., \& Rosales, G. (2015). Uma alternativa para a extração de lítio de pegmatitos. Anais Do XXVI Encontro Nacional De Tratamento De Minérios; 2015, 1(1).

Garrett, D. (2004). Handbook of lithium and natural calcium chloride: their deposits, processing, uses and properties (1st ed.). Amsterdam: Elsevier Academic.

Habashi, F. (1997). Handbook of Extractive Metallurgy (1st ed.).

Jeppson, D., Ballif, J., Yuan, W., \& Chou, B. (1978). Lithium literature review: Lithium's properties and interactions.

Lide, D. (1985). Handbook of Chemistry and Physics (1st ed.). Nova York.

MEDINA, L., \& El-Naggar, M. (1984). Handbook of Extractive Metallurgy (15th ed., pp. 725-726). Metallurgical Transactions.

Moreno, L. (2013). A strategic energy metal. Significant increase in demand ahead. Euro Pacific Canada. 